\title{
Ways of application of the provisions of mechanics of bodies with cracks to the calculation of asphalt concrete on strength and plasticity
}

\author{
Anatoly Aleksandrov ${ }^{1}$, Natalya Aleksandrova $^{1}$, Vasiliy Chusov ${ }^{1 *}$, and Aleksandr Riabov ${ }^{1}$ \\ ${ }^{1}$ Siberian State Automobile and Highway University (SibADI), pr. Mira 5, Omsk, 644080, Russia
}

\begin{abstract}
The report discusses the principles of two major theories of fracture mechanics of bodies with cracks, which include the theory of accumulation of damage Kachanov-Rabotnov and theory of brittle fracture Griffith-Irwin, including the invariant integral Cherepanov-Rice, describing the criterion of growth the crack. To assess the application of these theories to the calculation of asphalt concrete, laboratory test data are given and based on their analysis the appropriate conclusions.
\end{abstract}

\section{Introduction}

In practice of design of non-rigid pavement type with asphalt concrete coating and the base is required to check the strength of the lower layer by the criterion of resistance to fatigue fracture during bending [1]. This criterion applies the first strength theory, according to which the maximum normal horizontal stress should not exceed the fatigue strength of asphalt concrete in the lower layer of the coating or base. The calculation of normal tensile stresses is performed according to the nomogram, which is a graphical interpretation of the M. B. Korsunsky solution [2], which is based on the linear theory of elasticity. The disadvantage of the normative method of calculation can be considered:

1. Application of the first theory of strength, according to which impact on strength of other components of the stress tensor is not taken into account. It follows that when applied to the calculation of asphalt concrete pavement theory of bending of thin plates, only one tensile stress is introduced into the strength criterion, and the effect of the second tensile stress which weakening the first one is not taken into account. Therefore, the first strength theory underestimates the strength of asphalt concrete in the coating or base. According to experts in the field of phenomenological theories of strength, the criterion of the maximum normal stresses has a purely historical significance [3].

2. For the calculation of fatigue strength the fatigue function is applied, according to which the asphalt concrete in bending can withstand any number of loads. This contradicts the experimental data obtained during the testing of large-size asphalt concrete beams [4]

3. Calculation scheme of the road pavement is a two-layer construction in which the stress state differs from the stress state of the multi-layer pavement. For example, abroad for

\footnotetext{
*Corresponding author: chysow@gmail.com
} 
the calculation of road pavements with asphalt concrete layers software products are used, which are based on the solutions of the elasticity theory of the multilayer environment or numerical methods [5] (table 1).

From the data analysis of table 1 it follows, that in addition to improving the normative method of calculation [1] in terms of the shortcomings noted by us, relevant the work, aimed at taking into account the influence of friction forces and the development of a technique to calculate the asphalt concrete layers of shear resistance and accumulation of plastic deformation.

Table 1. Software products used abroad for the calculation of pavement

\begin{tabular}{|c|c|c|c|c|}
\hline Program & Theoretical basis & $\begin{array}{l}\text { Number } \\
\text { of layers }\end{array}$ & Developer & Note \\
\hline $\begin{array}{c}\text { BISAR } \\
{[6]}\end{array}$ & $\begin{array}{c}\text { The theory of linear } \\
\text { elasticity }\end{array}$ & 5 & Shell international & $\begin{array}{l}\text { The predecessor of this } \\
\text { product is the BISTRO } \\
\text { program }\end{array}$ \\
\hline $\begin{array}{c}\text { ELSYM } \\
{[7]}\end{array}$ & $\begin{array}{c}\text { The theory of linear } \\
\text { elasticity }\end{array}$ & 5 & FHWA (UCB) & The program is widely used \\
\hline $\begin{array}{l}\text { PDMAP } \\
\text { (PSAD) }[8]\end{array}$ & $\begin{array}{c}\text { The theory of linear } \\
\text { elasticity }\end{array}$ & 5 & $\begin{array}{c}\text { NCHRP Project I- } \\
10\end{array}$ & $\begin{array}{c}\text { Includes an application that } \\
\text { takes into account } \\
\text { nonlinear deformation }\end{array}$ \\
\hline $\begin{array}{c}\text { JULEA } \\
{[9]}\end{array}$ & $\begin{array}{c}\text { The theory of linear } \\
\text { elasticity }\end{array}$ & 5 & USACE WES & $\begin{array}{c}\text { Used in the program } \\
\text { «LEDFAA» }\end{array}$ \\
\hline $\begin{array}{l}\text { CIRCLY } \\
{[10]}\end{array}$ & $\begin{array}{l}\text { The theory of linear } \\
\text { viscoelastic medium }\end{array}$ & $\begin{array}{l}5 \text { and } \\
\text { more }\end{array}$ & $\begin{array}{l}\text { MINCAD, } \\
\text { Australia }\end{array}$ & $\begin{array}{l}\text { Includes application for } \\
\text { horizontal loads (friction } \\
\text { accounting) }\end{array}$ \\
\hline VESYS [11] & $\begin{array}{l}\text { The theory of linear } \\
\text { elasticity or } \\
\text { viscoelastic medium }\end{array}$ & 5 & FHWA & $\begin{array}{c}\text { Can be used to calculate } \\
\text { elastic or viscoelastic } \\
\text { media }\end{array}$ \\
\hline VEROAD [12] & $\begin{array}{l}\text { The theory of linear } \\
\text { elasticity or } \\
\text { viscoelastic medium }\end{array}$ & 15 & TU Delft & $\begin{array}{l}\text { Allows you to calculate the } \\
\text { plastic shear strain }\end{array}$ \\
\hline ILLIPAVE [13] & Finite element method & - & UIC & No features \\
\hline FENLAP [14] & Finite element method & - & $\begin{array}{l}\text { University of } \\
\text { Nottingham }\end{array}$ & $\begin{array}{c}\text { Allows to take into account } \\
\text { nonlinear properties of } \\
\text { materials }\end{array}$ \\
\hline
\end{tabular}

The calculation of plastic deformation and shear strength should be based on the appropriate plasticity conditions, which should take into account all components of the stress tensor.

To account for friction, it is necessary to improve the normative methodic, so that it is possible to calculate the components of the stress tensor at any point in the half-space, and not only at the points located on the axis of symmetry of the load. This requirement is due to the fact that in classical solutions of the theory of elasticity, the load applied to the surface of the half-space compresses this half-space before itself and stretches it beyond the point of application of force.

Therefore if load is applied in the center of the wheel imprint, then in the direction of the action of the force the half-space contracts, and in the direction of the opposite action of the force is stretched. The points of the layered half-space, located on the axis of symmetry of the distributed load, with respect to friction remain at rest.

Of course, that we have noted a large number of shortcomings of the technique [1] which requires the division of the problem of improving the calculation of asphalt pavement on a number of smaller problems and their serial solution. 


\section{Materials and Methods}

In this paper, the authors aim to assess the possibility of application for the calculation of asphalt concrete layers on the strength and plasticity, the theories of fracture mechanics of bodies with cracks. The objectives of the publication are:

- theoretical substantiation of possibility of transformation of criteria of durability and conditions of plasticity of continuous media in the criteria of calculation on durability and plasticity of bodies with cracks;

- test of asphalt samples for the determination of the measures of theory of damage and the theory of cracks corresponding to peak strength.

To solve the first problem, we consider the main provisions of the theory of damage accumulation in an environment that perceives an external load. The foundations of this theory are laid By L. M. Kachanov [15] and Y. N. Rabotnov [16], they proposed to use in the calculations of bodies with structure defects two measures varying from 1 to 0 (continuity) and from 0 to 1 (damage). The first quantity is called continuity, it was suggested to apply L. M. Kachanov [15]. This measure is understood as the ratio of stresses in a continuous $\sigma$ and damaged material $\sigma_{D}\left(\sigma_{D} \geq \sigma\right)$. The second measure was called damage, it was proposed by Y. N. Rabotnov [16]. Under the damage is the ratio of the sum cross-sectional area of the sample, occupied by defects $F_{D}$ to the geometric area of the whole, not destroyed section $F$ $\left(F_{D} \leq F\right)$. The wording recorded by us in words that have a mathematical expression, according to which measures of the theory of damage find by the formulas:

$$
\psi=\frac{\sigma}{\sigma_{D}} ; \quad \omega=\frac{F_{D}}{F} .
$$

It is found that both measures are related to each other, and their relationship can be expressed through the formula:

$$
\omega+\psi=1 ; \quad \omega=1-\psi ; \quad \psi=1-\omega .
$$

From the analysis of the first formula from the dependencies (2) it follows that the sum of the two measures is equal to one, that is, the sum of these damage and continuity is nothing but the geometric cross-sectional area expressed as unit. According to two other expressions of formulas (2), the value of one parameter of the theory of stress accumulation is calculated as the difference between one and the second parameter through the value of another characteristic.

Hypothesis L. M. Kachanov, written as the first expression (1) can be applied to any component of the stress tensor, and applying the third expression of formulas (2) components of the stress tensor of the body, affected by defects $\sigma_{\mathrm{ijD}}$, can be determined through the components of the stress tensor of a solid body $\sigma_{\mathrm{ij}}$ and damage. That is, by formulas

$$
\sigma_{i j D}=\frac{\sigma_{i j}}{1-\omega}=\frac{\sigma_{i j}}{\psi} \text {. }
$$

Value of measures of the theory of accumulation is determined by the methods developed by experts in the field of mechanics of damaged media [17]. One of such methods is the principle of equivalence of deformations. According to this principle, the formulas are valid:

$$
E_{D}=E \cdot(1-\omega) ; \quad \omega=1-\frac{E_{D}}{E} ; \quad \psi=\frac{E_{D}}{E} .
$$

where $E$ and $E_{D}$ - elastic modulus (Young's) of undamaged and damaged material, Pa. 
Of course, any characteristic of the stress tensor of the damaged body can be determined through the stress components of this body. For example, the dependence (3) allows to calculate the main stresses in the damaged medium through the corresponding components of the stress tensor of a solid body. After determining these characteristics, octahedral stresses, Mises intensities, and any invariant of the stress tensor and stress deviator can be calculated. These invariant characteristic can be used in the criteria of strength and plasticity conditions. In [18] we modified the strength criteria of Mohr and Pisarenko-Lebedev so that these criteria contain either continuity or damage. For the modification of the conditions of plasticity, analyzed the conditions of plasticity is obtained in the works $[19,20]$. This is the condition of plasticity is a modification of the criterion of Coulomb-Mohr performed by the input of the third material parameter $d$. Depending on the value of this parameter, the criterion allows to cover the range of variation of tangential stress from the value, corresponding to the original Coulomb-Mohr criterion (at $\mathrm{d}=0.5$ ) up to the value corresponding to the limit state condition according to the third strength theory (at $\mathrm{d}=0$ ). The modified criterion of plasticity is given in the paper [21]. Modification of criteria of Mohr, Pisarenko-Lebedev and three-parameter condition Coulomb-Mohr are shown in table 2, these criteria can be applied in the calculation of asphalt concrete layers for flexural strength and shear strength in plastic deformation.

Table 2. Modified criteria, including the measures of theory of damage

\begin{tabular}{|c|c|}
\hline $\begin{array}{c}\text { The name of the original } \\
\text { criterion }\end{array}$ & $\begin{array}{c}\text { Conditions of the limit state of the modified criterion including } \\
\text { damage }\end{array}$ \\
\hline $\begin{array}{c}\text { The strength criterion } \\
\text { Mohr }\end{array}$ & $\left(\sigma_{1}-\frac{R_{\mathrm{st}}}{R_{\mathrm{com}}} \cdot \sigma_{3}\right) \cdot \frac{1}{1-\omega}=R_{\mathrm{st}}$ \\
\hline $\begin{array}{c}\text { Criterion of plasticity of } \\
\text { Pisarenko-Lebeder }\end{array}$ & $\frac{1}{1-\omega} \cdot\left[\chi \cdot \sigma_{\mathrm{int}}+(1-\chi) \cdot \sigma_{1}\right]=R_{\mathrm{st}} ; \quad \chi=R_{\mathrm{st}} / R_{\mathrm{com}}$ \\
\hline $\begin{array}{c}\text { Three- parameter } \\
\text { Coulomb-Mohr condition }\end{array}$ & $\frac{1}{2 \cdot(1-\omega)} \cdot\left(\sigma_{1} \cdot\left(\frac{1-\sin \varphi}{1+\sin \varphi}\right)^{d}-\left(\frac{1+\sin \varphi}{1-\sin \varphi}\right)^{d} \cdot \sigma_{3}\right)=c$ \\
\hline
\end{tabular}

Note: $\sigma_{1}$ and $\sigma_{3}-$ the maximum and minimum principal stresses in the intact body, $\mathrm{Pa}$; $\sigma_{\text {int }}-$ the intensity of normal stresses in a solid body, $\mathrm{Pa} ; R_{\text {com }}$ and $R_{\mathrm{st}}-$ strength limits of uniaxial compression and tensile from bending, $\mathrm{Pa} ; \chi$ - the coefficient of plasticity of the material, characterizing the degree of responsibility for the micro-destruction of shear deformation, creating favorable conditions for loosening the material and cracking.

Another direction in the calculations of bodies with defects of the structure is brittle fracture mechanics, which developed by A. Griffith and G. Irwin. Currently, the most wellknown criteria of the crack theory are the crack growth criteria obtained on the basis of invariant integrals G.P. Cherepanov [22] and J.R. Rice [23]. Criteria G.P. Cherepanov and J.R. Rice recorded in the form:

$$
\mathrm{G}=\mathrm{G}_{\mathrm{c}} ; \quad \mathrm{G}=f\left(K_{\mathrm{I}}, K_{\mathrm{II}}, K_{\mathrm{III}}\right) ; \quad J=J_{\mathrm{c}} ; \quad J=f\left(K_{\mathrm{I}}, K_{\mathrm{II}}, K_{\mathrm{III}}\right),
$$

Where $K_{I}, K_{I I}$ и $K_{I I I}$ - the stress intensity factors for cracks I, II and III-rd types of cracks respectively, $\mathrm{G}$ and $J$-the actual values of the G-integral of G.P. Cherepanov and $J$-integral of J.R. Rice; $\mathrm{G}_{\mathrm{c}}$ and $J_{c}$ - critical values of the corresponding invariant integrals, also called "elastoplastic fracture toughness".

The connection of $J$-integral with stress intensity factor for cracks of the I-th and mixed types J.R. Rice showed by addictions [23, p. 381]. In general the $J$-integral is given by formula: 


$$
J=\frac{1-\mu^{2}}{E} \cdot\left(K_{\mathrm{I}}^{2}+K_{\mathrm{II}}^{2}\right) \cdot \frac{1+\mu}{E} \cdot K_{\mathrm{III}}^{2} .
$$

The right part of the equation (6) is the rate of energy release during crack growth in an elastic body [5]. Critical value $J_{c}$ determine experimentally. In the future, when solving the problems of fracture mechanics, the $J$-integral was the sum of two components: elastic $J_{e}$ and plastic $J_{p}$.

To asphalt concrete pavement Cherepanov - Rice criterion applied by A.A. Malyshev [24]. A.A. Malyshev proposed a characteristic of crack resistance of asphalt concrete under the influence of cyclic loads, called the index of fracture toughness. An experimental technique has been developed to determine this characteristic [24, item 2]. As a result, A.A. Malyshev obtained the power-law dependence of the fracture toughness index on the number of applied design loads. The A.A. Malyshev model contains two parameters of material, set for fine-grained dense and porous as well as dense sand asphalt concrete. In addition, it was found that the period of crack growth to the critical size corresponds to a certain value of the accumulated plastic deformation $\varepsilon_{\mathrm{p}}$. This deformation serves as the second criterion of the limit state, which is understood as the onset of the moment of exhaustion of the plastic potential and the increase in the crack to a dangerous size [24, p. 25].

\section{Results}

To assess the applicability of the theory of damage and crack accumulation to the calculation of asphalt concrete layers, it is necessary to have experimental data on the kinetics of damage accumulation and crack growth. For these purposes, we have made cylindrical samples, which were tested by uniaxial compression. The diameter of each sample was $10 \mathrm{~cm}$, and the total number of 12 . The samples are divided into three groups, each of which was tested at a certain temperature. The first group of 4 samples was tested at a material temperature of 10 degrees Celsius, the second group, including 4 samples, tested at an asphalt temperature of 25 degrees Celsius, and the third group - 50 degrees Celsius. The samples were thermostated in the chamber heat and cold, after which the sample was tested by compression, setting the test program on the computer. Preparation for the test involves entering the initial data, which are selected as the type of test (uniaxial compression), units of measurement of pressure and strain and enter information about the sample (diameter and height) and the conditions of the experiment (the value of the ultimate deformation, strain rate). After that, the test is run, which is performed with the specified parameters until the specified value of the limit deformation or complete destruction of the sample, if it occurs when the deformation is less than the limit value.

To calculate the damage, the principle of equivalence of deformations was applied, which is written by the second expression of formulas (4), with the difference that the deformation modules of a solid body and a damaged medium were determined by a special technique. To do this, the test results were given by graphical ratio of deformation from stress. On such graphic dependences the straight line segment was clearly seen, within which the linear dependence between deformation and pressure acts. Along the pressure axis, the straight line segment is limited by the value of the proportionality limit $p_{\mathrm{p}}$. Therefore, to the dependence of the strain on the pressure, the value of which is in the range $0 \leq p_{\mathrm{p}} \leq p$ apply Hooke's law of proportionality. From this law, described by the linear dependence of strain and pressure, the deformation modulus of an intact body was calculated $E$. If the pressure applied to the sample exceeds the proportionality limit, the relationship of this pressure with the deformation is nonlinear. The nonlinear sector of the graph is replaced by a piecewise linear dependence. The nodes (points) of such a piecewise linear dependence were the measured pressures and the corresponding deformations. For each interval of such dependence the deformation 
modulus was determined. Since the considered area of dependence from pressure is nonlinear, the calculated module can be considered as the elastic-plastic deformation module. For each section of the piecewise linear dependence, the elastic-plastic deformation modulus was calculated, which was considered the deformation modulus of the damaged medium $E_{D}$. Such modules are calculated for the section of the curve from the proportionality limit $p_{\mathrm{p}}$ to the limit of the compressive strength $R_{\text {com. }}$.

As a measure of the stress state, are taken difference between pressure and the proportionality limit. Given that the pressure on the sample is equal to the normal vertical tension $\sigma_{z}$ in an intact body, a measure of the stress state can be recorded in the form of:

$$
p-p_{p}=\sigma_{z}-p_{p}
$$

Figure 1 shows the dependence of the damage of asphalt concrete sample from the measure of stress state with characterized by the difference (7).

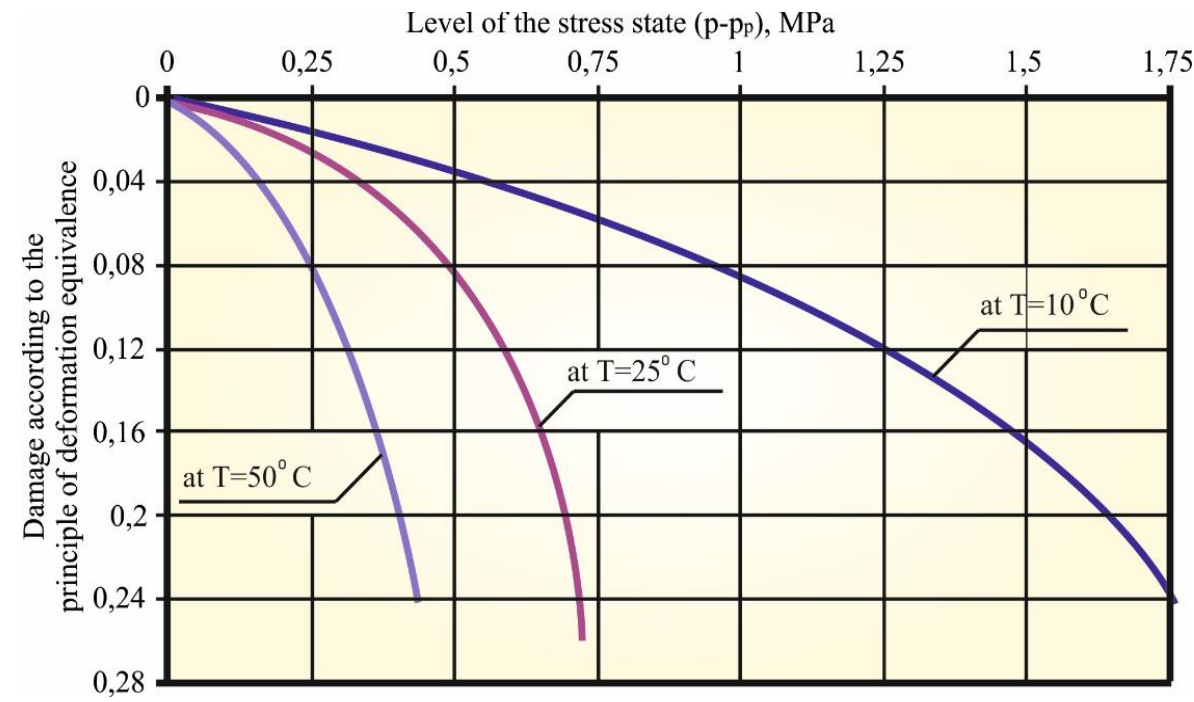

Fig. 1. Dependence of damage from the level of stress state $\sigma_{z}-p_{p}$

Table 3. shows the value damage, calculated by the second dependence of the formulas (4) and the corresponding stress state levels which calculated by the expression (7).

Table 3. Results of tests of asphalt concrete samples by compression

\begin{tabular}{|c|c|c|c|c|c|c|c|c|c|}
\hline 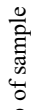 & $\begin{array}{l}\text { 芯 } \\
\text { : } \\
\text { : }\end{array}$ & \multicolumn{8}{|c|}{ The value of damage and the level of stress state } \\
\hline \multirow[b]{2}{*}{1} & \multirow[b]{2}{*}{$10^{\circ} \mathrm{C}$} & $\sigma_{z}-\mathrm{p}_{\mathrm{p}}, \mathrm{MPa}$ & 0,25 & 0,50 & 0,75 & 1,00 & 1,25 & 1,50 & 1,75 \\
\hline & & $\begin{array}{c}\omega, \text { percentage of } \\
\text { unit }\end{array}$ & 0,016 & 0,036 & 0,058 & 0,085 & 0,120 & 0,166 & 0,240 \\
\hline \multirow[b]{2}{*}{2} & \multirow[b]{2}{*}{$25^{\circ} \mathrm{C}$} & $\sigma_{z}-\mathrm{p}_{\mathrm{p}}, \mathrm{MPa}$ & 0,125 & 0,25 & 0,375 & 0,5 & 0,57 & 0,65 & 0,72 \\
\hline & & $\begin{array}{c}\omega, \text { percentage of } \\
\text { unit }\end{array}$ & 0,016 & 0,026 & 0,049 & 0,082 & 0,113 & 0,160 & 0,260 \\
\hline \multirow[b]{2}{*}{3} & \multirow[b]{2}{*}{$50^{\circ} \mathrm{C}$} & $\sigma_{z}-\mathrm{p}_{\mathrm{p}}, \mathrm{MPa}$ & 0,063 & 0,125 & 0,188 & 0,25 & 0,31 & 0,38 & 0,44 \\
\hline & & $\begin{array}{c}\omega, \text { percentage of } \\
\text { unit }\end{array}$ & 0,012 & 0,029 & 0,052 & 0,083 & 0,118 & 0,170 & 0,240 \\
\hline
\end{tabular}


Table 4 shows the mathematical models, describing the data of tables 1-3, and the coefficients of determination, which are an estimate of the accuracy of the empirical formula.

Table 4. Power-law mathematical models of damage

\begin{tabular}{|c|c|c|c|}
\hline \multirow{2}{*}{$\begin{array}{c}\text { Sample } \\
\text { temperature, }\end{array}$} & \multicolumn{3}{|c|}{ Mathematical model and determination coefficient } \\
\cline { 2 - 4 } & Description & Formula & Estimate $\mathrm{R}^{2}$ \\
\hline 10 & Power-law & $\omega=0,094 \cdot\left(\sigma_{z}-p_{p}\right)^{1,352}$ & 0,987 \\
\hline 25 & Power-law & $\omega=0,286 \cdot\left(\sigma_{z}-p_{p}\right)^{1,534}$ & 0,924 \\
\hline 50 & Power-law & $\omega=0,726 \cdot\left(\sigma_{z}-p_{p}\right)^{1,518}$ & 0,992 \\
\hline
\end{tabular}

\section{Conclusions}

Analysis of the test data shows that the value of the peak strength, which in figure 1 is the far right point, corresponds to a certain value of damage. This value is less than 1 , which means when load on the sample corresponds to the strength of the material on uniaxial compression, the sample is not completely destroyed. The complete destruction, the damage equal to 1 , and continuity -0 . Therefore, the application of the principles of the theory of damage to the calculation of asphalt requires both mathematical modeling of damage from the magnitude of the load, the number of their applications, the time of one load, the temperature of the asphalt concrete layer and other parameters of the material, and determining the final value of damage corresponding to the peak strength of the material. Regardless of the test temperature, the final damage value is almost the same for each of the three groups of tested samples. This value is in the range from 0,24 to 0,26 . Therefore, the final value of the damage can be considered as a certain limit parameter of the material characterizing its peak strength.

On the other hand, the almost invariable damage value at the pressure corresponding to the compressive strength confirms the fact that for each group of samples there is a critical crack length at which the fracture occurs. This leads to the conclusion that, in contrast to the peak strength, the corresponding values of damage and crack length are constants that uniquely determine the conditions of material failure.

\section{References}

1. ODN 218.046-01. Design of non-rigid road clothes (GSDH of Ministry of Transport, Moscow, 2001)

2. N.N. Ivanov Construction and calculation of non-rigid pavement (Transport, Moskow, 1973)

3. I.I. Gol'denblat, V.A. Kopnov Criteria of strength and plasticity of structural materials (Engineering, Moscow, 1968)

4. G.V. Levashov Improvement of the method of calculation of reinforced asphalt concrete coatings and bases according to the fatigue failure criterion (Ph.D. thesis)

5. C. Monismith Ten Year Perspective on Accelerated Pavement Testing; Caltrans Partnered Pavement Research Program (Proceedings of the 2nd International Conference on Accelerated Pavement Testing, Minneapolis Sep. 26-29, 2004)

6. D.L. de Jong, M.G.F. Peutz, A.R. Korswagen Computer Program BISAR: Layered Systems Under Normal and Tangential Loads (External Report AMSR.0006.73, Koninklijke Shell-Laboratorium, Amsterdam, 1973) 
7. G. Ahlborn, ELSYM5 Computer Program for Determining Stresses and Deformations in Five Layer Elastic Systems (University of California, Berkeley, 1972)

8. E.L. Skok, F.N. Finn Theoretical Concepts Applied to Asphalt Concrete Pavement Design (International Conference on the Structural Design of Asphalt Pavements, University of Michigan, 1963)

9. J. Uzan, Influence of the Interface Condition on Stress Distribution in a Layered System (Transportation Research Board, Washington, 1976)

10. L.J. Wardle Program CIRCLY, A Computer Program for the Analysis of Multiple Complex Circular Loads on Layered Anisotropic Media (Division of Applied Geomechanics, Commonwealth Scientific and Industrial Research Organization, Victoria, 1977)

11. W.J. Kenis Predictive Design Procedures, VESYS User's Manual: An Interim Design Method for Flexible Pavements Using the VESYS Structural Subsystem (Final Report FHWA-RD-164, Federal Highways Administration, U.S. Department of Transportation, Washington, 1978)

12. R.N. Nilsson, I. Oost, P.C. Hopman Viscoelastic Analysis of Full-Scale Pavements: Validation of VEROAD (Transportation Research Record 1539, Transportation Research Board, Washington, 1996)

13. M.R. Thompson, R.P. Elliott ILLI-PAVE Based Response Algorithms for Design of Conventional Flexible Pavements (Transportation Research Record 1207, Transportation Research Board, National Research Council, Washington, 1988)

14. J.M. Brunton, J.R. d'Almeida Modeling Material Non-Linearity in a Pavement BackCalculation Procedure (Transportation Research Record 1377, Transportation Research Board, National Research Council, Washington, 1992)

15. L.M. Kachanov Fundamentals of fracture mechanics (Science, Moscow, 1974)

16. U.N. Rabotnov Mechanics of deformable solids (Science, Moscow, 1979)

17. J. Lemaitre A Course on Damage Mechanics (Verlag Berlin Heidelberg: Springer, 2005)

18. N.P. Aleksandrova, V.V. Chysow Magazine of Civil Engineering №2 69 (2016)

19. A.S. Aleksandrov, A.L. Kalinin Engineering and construction magazine №7 4 (2015)

20. A.S. Aleksandrov, G.V. Dolgih, A.L. Kalinin Engineering and construction magazine №2 51 (2016)

21. N.P. Aleksandrova, A.S. Aleksandrov, V.V. Chusov Application of Principles of Theory of Damage Accumulation to Calculation of Asphalt-Concrete Coatings (IOP Conf. Ser.: Mater. Sci. Eng. Vol. 262. 2017)

22. G.P. Cherepanov Applied Mathematics and Mechanics t31, №3, 476 (1967)

23. J.R. Rice Journal of Applied Mechanics vol 35379 (1968)

24. A.A. Malyshev Forecasting residual strength strength of road clothes with asphalt concrete pavements (SibADI, Omsk, 2004) 